\title{
Variable Range Hopping Conduction in Low-Temperature Molecular Beam Epitaxy GaAs
}

\author{
R. M. Rubinger*, H. A. Albuquerque ${ }^{\dagger}$, R. L. da Silva ${ }^{\dagger}$, A. G. de Oliveira $^{\dagger}$, \\ G. M. Ribeiro ${ }^{\dagger}$, W. N. Rodrigues ${ }^{\dagger}$, C. P. L Rubinger*, and M. V. B. Moreira ${ }^{\dagger}$ \\ *Departamento de Física e Química, Instituto de Ciências, \\ Universidade Federal de Itajubá, Caixa Postal 50, 37500-903, Itajubá, Brazil and \\ ${ }^{\dagger}$ Departamento de Física, Instituto de Ciências Exatas, \\ Universidade Federal de Minas Gerais, Caixa Postal 702, 30123-970, Belo Horizonte, Brazil
}

Received on 4 April, 2005

\begin{abstract}
Electric transport properties measured by Van der Pauw resistivity experiments of Low-Temperature Molecular Beam Epitaxy (LT-MBE) GaAs samples are used to identify a method to improve the resistivity of GaAs material. We present results on five samples grown at $265,310,315,325$, and $345^{\circ} \mathrm{C}$. The electric measurements were carried out at temperatures ranging from 130 to $300 \mathrm{~K}$. In this temperature range the dominant transport process is identified as variable range hopping. The hopping parameter plotted against the growth temperature is shown to present a maximum. The mechanisms responsible for this behavior are discussed in relation to the compensation ratio.
\end{abstract}

Keywords: GaAs; Variable range hopping; Resistivity measurements

\section{INTRODUCTION}

The search of GaAs layers grown by MBE with good quality semi-insulating (SI) properties has been pursued for at least two decades [1] and it can be improved by the control of intrinsic defect density through the growth temperature.

The SI characteristic is related to the presence of intrinsic deep level defects [2]. Although deep levels leads to recombination, in high enough density it also transport carriers by hopping. This is an undesirable characteristic. In the present work we varied the growth temperature in an attempt to maximize the resistivity.

Albeit its technological implications, from the academic point of view, the study of hopping conduction of deep level defects on GaAs offers a fruitful platform for the study of hopping conduction at temperatures as high as the room temperature $[3,4]$. Previous reports on the literature have identified hopping conduction among arsenic antisite defects $\left(\mathrm{As}_{\mathrm{Ga}}\right)$ in GaAs $[3,4]$. We present here our results for resistivity measurements in five LT-MBE GaAs samples. The contributions of the present work concern only to the Variable Range Hopping (VRH).

Temperature dependent Van der Pauw resistivity measurements for the five samples show VRH signature associated with the law $\rho=\rho_{0} \exp \left(T_{0} / T\right)^{1 / 4}$ over a wide temperature range. The parameter $T_{0}^{1 / 4}$ is an indirect measurement of the density of centers participating in hopping conduction and is a signature of the VRH. It is observed that for increasing growth temperature $\left(\mathrm{T}_{G}\right)$ the hopping parameter presents a maximum at $315^{\circ} \mathrm{C}$. Such feature is associated with a low compensation ratio. Another important issue concerns the trapping characteristics of deep level defects in the temperature range considered in this work. This makes negligible any contribution of shallow extrinsic impurities and of free carriers as indicated by pure VRH.

\section{EXPERIMENTAL DETAILS AND RESULTS}

In the present work we report on VRH in LT-MBE GaAs samples grown at $265,310,315,325$, and $345^{\circ} \mathrm{C}$. The LTGaAs layers were grown on (100) semi-insulating LEC GaAs substrates, in a RIBER 2300 MBE system. After the standard desorption procedures a buffer layer $0.21 \mu \mathrm{m}$ thick was grown at $610^{\circ} \mathrm{C}$ under an As overpressure of $3.2 \times 10^{-5}$ Torr and 0.81 monolayer/s. After that the substrate temperature was ramped down to the final desired temperature at $10{ }^{\circ} \mathrm{C} / \mathrm{min}$ while growing. After reaching the set temperature GaAs epilayers were grown having around $2.4 \mu \mathrm{m}$ thick.

Microprobe analyses were carried out in order to determine the excess arsenic of the samples by comparing them with a standard GaAs wafer. The measurements were done in a JEOL JXA-8900RL in the wavelength dispersive mode considering 10 different regions per sample, $1 \mu \mathrm{m}$ diameter spots, an acceleration voltage of $25 \mathrm{kV}$, and a counting time of $10 \mathrm{~s}$. The excess arsenic as a function of the growth temperature are listed in Table I.

TABLE I: Hopping parameters

\begin{tabular}{|l|l|l|l|}
\hline Sample \# $\left(\mathrm{T}_{G}^{o} \mathrm{C}\right)$ & $\begin{array}{l}\text { Excess } \\
\mathrm{As}(\%)\end{array}$ & $T_{0}^{1 / 4}\left(\mathrm{~K}^{1 / 4}\right)$ & $N_{h}\left(\mathrm{~cm}^{-3}\right)$ \\
\hline $1(265)$ & 1.95 & $99.0 \pm 0.5$ & $(4.7 \pm 0.1) \times 10^{19}$ \\
\hline $2(310)$ & 1.80 & $190 \pm 1$ & $(9.3 \pm 0.1) \times 10^{18}$ \\
\hline $3(315)$ & 1.77 & $370 \pm 2$ & $(1.7 \pm 0.1) \times 10^{16}$ \\
\hline $4(325)$ & 1.84 & $260 \pm 1$ & $(1.4 \pm 0.1) \times 10^{18}$ \\
\hline $5(345)$ & 1.71 & $232 \pm 1$ & $(2.8 \pm 0.1) \times 10^{18}$ \\
\hline
\end{tabular}

For the resistivity van der Pauw measurements the samples were cut into $5 \times 5 \mathrm{~mm}^{2}$ squares and the contacts were made of indium drops. The samples were then baked at $250{ }^{\circ} \mathrm{C}$ for 10 min under flowing inert gas. The measurements were carried out in the "ohmic" regime (i.e. under applied bias which respect the linearity of the ohm's law). The ohmic range of 
the applied bias was previously identified from I(V) conductivity measurements, which were carried out from negative to positive bias.

For each growth temperature, we have prepared at least seven samples from different parts of the wafer and the results did not vary significantly in a same wafer. For the resistivity measurements, we have used a Keithley electrometer model 6517 and a current/voltage source Keithley model 237. Both instruments were used with triaxial cables in guarded-mode measurements. The electric transport measurements were carried out at temperatures ranging from 130 to $300 \mathrm{~K}$.

Resistivity measurements in a logarithmic scale as a function of $T^{-1 / 4}$ are shown in Fig. 1. Standard VRH (variable range hopping) corresponds to the range of linear dependence of measurements in this diagram [5]. The values of $T_{0}^{1 / 4}$ obtained by fitting this linear region are listed in Table I.

\section{DISCUSSION}

According to Mansfield [6] the active center density carrying Variable Range Hopping $(\mathrm{VRH})-$ denoted $N_{h}-$ can be obtained from $T_{0}$ (in $\mathrm{K}$ ) combining the following expressions:

$$
T_{0}=\frac{49}{k N_{0} a^{3}},
$$

and

$$
N_{0}=\frac{\kappa N_{h}^{2 / 3}}{e^{2}}
$$

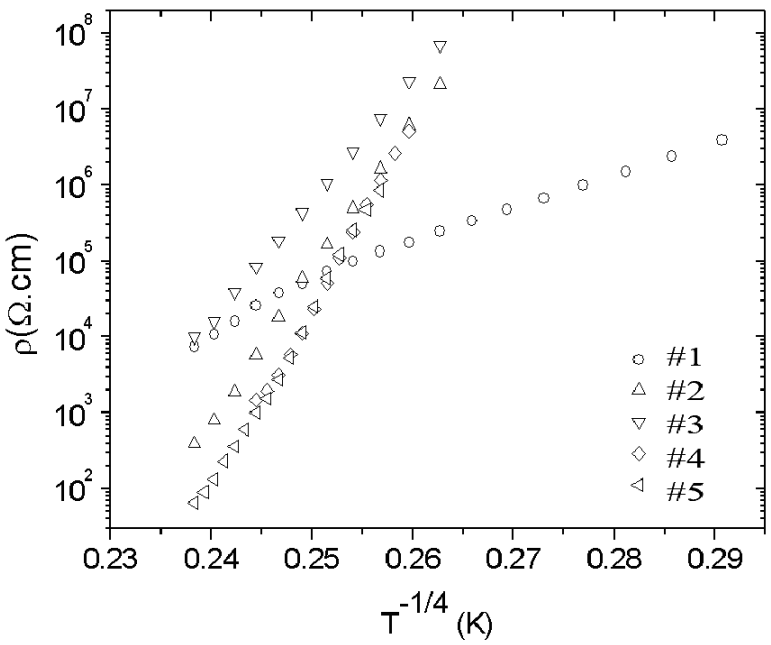

FIG. 1: VRH characteristic resistivity plot versus $T^{-1 / 4}$.

In Eq. (1) factor 49 is the percolation parameter, $\mathrm{k}$ is the Boltzmann's constant and $a$ is the wave function localization radius of the hopping active center. The value of $a$, which estimated the localization radius of the defect, was estimated to be $4 \AA$ from X-ray diffraction experiments by Shimoghishi et. al. [7]. Finally, Eq. (2) relates $N_{0}$ to $N_{h}$, where the dielectric constant $\kappa$ is assumed to be $12.9 \epsilon_{0}$ for GaAs [3] (where $\epsilon_{0}$ is the vacuum dielectric constant) and $e$ is the electron charge. $N_{0}$ is directly related to the density of states associated with the $\operatorname{As}_{G a}$ [6]. It should be noted that $N_{h}<N_{D}$, i.e., $N_{h}$ is always smaller than $N_{D}$, which is the $\mathrm{As}_{G a}$ donor density or any other defect responsible for the VRH effect. Since $N_{h}$ values were obtained from $T_{0}^{1 / 4}$ which is obtained by linear fittings of Fig. 1 its value holds for the temperature range considered in the fittings.

The hopping conduction involves only the energy levels within a narrow range near the Fermi level, and this range shrinks as the temperature is reduced [8]. By decreasing the temperature, the active defect density for hopping conduction also decreases. The shrinking of the narrow energy range is the key mechanism behind theexp $\left(T_{0} / T\right)^{1 / 4}$ law for VRH.

In Table I, besides the excess arsenic and the $T_{0}^{1 / 4}$ values, we listed the $N_{h}$ values calculated using Eq.(1) and Eq.(2). The ionization energy of the defect center was $0.65 \mathrm{eV}$ [4].

In this work we improved substrate characteristics by adjusting the growth temperature and reducing $N_{h}$. Since microprobe measurements indicates an almost constant excess arsenic which implies in low $\mathrm{As}_{G a}$ density changes. The reduction in $N_{h}$ can be associated with the compensation factor [6].

Based on the results presented in Table 1, the main outcomes can be summarized as follows. The order of magnitude of $N_{h}$, derived from resistivity measurements and Eq.(1) and Eq.(2), decreases from $10^{19} \mathrm{~cm}^{-3}$ for sample $\# 1$ to $10^{18} \mathrm{~cm}^{-3}$ for sample \#2 and further to a minimum at $10^{16} \mathrm{~cm}^{-3}$ for sample \#3. The sample \#3 presents the lowest $N_{h}$ value and for further increasing $T_{G}, N_{h}$ increases back to $10^{18} \mathrm{~cm}^{-3}$. A minimum in $N_{h}$ must be related to a low compensation levels and not to a real decrease in the $\mathrm{As}_{G a}$ density.

The microprobe analysis indicates excess arsenic between $1.71 \%$ and $1.95 \%$. In first approximation it can be considered constant, when compared with the huge significant change in the hopping active defects density $N_{h}$ (namely, three orders of magnitude). The $\mathrm{As}_{G a}$ defect density $\left(N_{D}\right)$ of sample \#3 is expected to be around $10^{19} \mathrm{~cm}^{-3}$, [9] only one order of magnitude lower than sample \#2 and the same order of the other samples. Another mechanism must be therefore considered in order to understand the reduction of the density of active centers supporting VRH. The $A s_{G a}^{+}$density depends directly on the acceptor density $N_{A}$. In LT-MBE GaAs the gallium vacancies $V_{G a}$ play the major role as acceptors [10]. This defect is known as a deep triple acceptor [7]. Regarding the $V_{G a}$ concentrations, Gebauer et al. [10] found densities of $10^{18} \mathrm{~cm}^{-3}$ and $10^{17} \mathrm{~cm}^{-3}$ for $T_{G}$ of $200{ }^{\circ} \mathrm{C}$ and $300{ }^{\circ} \mathrm{C}$, respectively.

Our work indicates that it is possible to obtain good quality semi-insulating GaAs epilayers required for applications in devices with low sidegating effects. We have shown that is possible to maximize the resistivity value by controlling the hopping parameter $T_{0}^{1 / 4}$ through the growth temperature. The presence of acceptors is another important experimental factor to be considered. Thus it is important to produce samples rich in AsGa but with negligible acceptor concentrations. Our LT- 
MBE samples grown at $315^{\circ} \mathrm{C}$ are highly resistive, presenting a higher hopping parameter $T_{0}^{1 / 4}$.

\section{SUMMARY}

We carried out high-precision van der Pauw resistivity measurements on five GaAs samples grown by low temperature molecular beam epitaxy (LT-MBE) at 265, 310, 315, 325, and $345{ }^{\circ} \mathrm{C}$. Our results show that the samples grown at $265^{\circ} \mathrm{C}$ present resistivity and hopping parameters similar to previous ones [3]. The other samples present higher hopping parameter with an extremum for the sample grown at $315^{\circ} \mathrm{C}$. This result could be understood in the framework of the Variable Range Hopping mechanism, and indicates that very high insulating materials can be obtained by LT-MBE, when the proper growth temperature window is chosen.

\section{Acknowledgements}

The compositional measurements were done in the Laboratório de Microscopia Eletrônica e Microanálise - LMA financed by FAPEMIG under contract CEX 1074/95.

We would like to thank K. Krambrock for the elucidative discussion. The authors acknowledge the Brazilian Agencies $\mathrm{CNPq}$ and FAPEMIG for financial support.
[1] J. M. Ballingall, Semiconductor Characterization, edited by W.M. Bullis, D.G. Seiler, and A.C. Diebold (AIP Press, New York, 1996), p. 578.

[2] D. C. Look, Thin Solid Films 231, 61 (1993).

[3] D. C. Look, D. C. Walters, M. O. Manasreh, J. R. Sizelove, C. E. Stutz, and K. R. Evans, Phys. Rev. B 42, 3578 (1990).

[4] D. C. Look, Z-Q. Fang, and J. W. Look, J. Electrochem. Soc. 141, 747 (1994).

[5] B. I. Shklovskii, Sov. Phys. Semic. 6, 1053 (1973).

[6] R. Mansfield, Hopping transport in solids, edited by M. Pollak and B.I. Shklovskii (North Holland, Amsterdam, 1991), p. 349.
[7] F. Shimogishi, K. Mukai, S. Fukushima, and N. Otsuka, Phys. Rev. B 65, 165311 (2002).

[8] B. I. Shklovskii and A. L. Efros, Electronic Properties of Doped Semiconductors (Springer Series in Solid-State Sciences, Springer-Verlag, Berlin, 1984) Vol. 45.

[9] K. Krambrock, M. Linde, J. M. Spaeth, D.C. Look, D. Bliss, and W. Walukiewicz, Semicond. Sci. Technol. 7, 1037 (1992).

[10] J. Gebauer, R. Krause-Rehberg, S. Eichler, M. Luysberg, H. Sohn, and E. R. Weber, Appl. Phys. Lett. 71, 638 (1997). 